\title{
Constraining the Minimum Mass of High-redshift Galaxies and Their Contribution to the Ionization State of the Intergalactic Medium
}

\section{Citation}

Muñoz, J. A., and A. Loeb. 2011. "CONSTRAINING THE MINIMUM MASS OF HIGH-REDSHIFT GALAXIES AND THEIR CONTRIBUTION TO THE IONIZATION STATE OF THE INTERGALACTIC MEDIUM." The Astrophysical Journal 729 (2): 99. https://doi.org/10.1088/0004-637x/729/2/99.

\section{Permanent link}

http://nrs.harvard.edu/urn-3:HUL.InstRepos:41393155

\section{Terms of Use}

This article was downloaded from Harvard University's DASH repository, and is made available under the terms and conditions applicable to Other Posted Material, as set forth at http:// nrs.harvard.edu/urn-3:HUL.InstRepos:dash.current.terms-of-use\#LAA

\section{Share Your Story}

The Harvard community has made this article openly available. Please share how this access benefits you. Submit a story. 


\title{
CONSTRAINING THE MINIMUM MASS OF HIGH-REDSHIFT GALAXIES AND THEIR CONTRIBUTION TO THE IONIZATION STATE OF THE INTERGALACTIC MEDIUM
}

\author{
J. A. MuÑOZ ${ }^{1,2}$ AND A. LOEB ${ }^{2}$ \\ ${ }^{1}$ University of California Los Angeles, Department of Physics and Astronomy, Los Angeles, CA 90095, USA \\ ${ }^{2}$ Harvard-Smithsonian Center for Astrophysics, 60 Garden Street, Cambridge, MA 02138, USA \\ Received 2010 October 12; accepted 2011 January 6; published 2011 February 14
}

\begin{abstract}
We model the latest $H S T$ WFPC3/IR observations of $\gtrsim 100$ galaxies at redshifts $z=7-8$ in terms of a hierarchical galaxy formation model with starburst activity. Our model provides a distribution of UV luminosities per dark matter halo of a given mass and a natural explanation for the fraction of halos hosting galaxies. The observed luminosity function is best fit with a minimum halo mass per galaxy of $10^{9.4_{-0.9}^{+0.3}} M_{\odot}$, corresponding to a virial temperature of $10^{4.9_{-0.7}^{+0.2}} \mathrm{~K}$. Extrapolating to faint, undetected galaxies, the total production rate of ionizing radiation depends critically on this minimum mass. Future measurements with JWST should determine whether the entire galaxy population can comfortably account for the UV background required to keep the intergalactic medium ionized.
\end{abstract}

Key words: cosmology: theory - early universe - galaxies: formation - galaxies: high-redshift - stars: formation

\section{INTRODUCTION}

The pursuit for the first galaxies has recently entered a new phase as observations at redshifts $z \gtrsim 6$ have now probed the epoch before cosmic reionization was complete enough to fill in the Gunn-Peterson trough. The WFC3/IR Camera on the Hubble Space Telescope (HST) has recently detected large samples of Lyman-break galaxies (LBGs) at $z=7$ and 8 (Bouwens et al. 2010b, 2010c; McLure et al. 2010; Bunker et al. 2010; Yan et al. 2010; Finkelstein et al. 2010) and provided constraints on the galaxy abundance as early as $z \sim 10$ (Bouwens et al. 2009). The UV spectral slopes of these faint sources were found to be very flat, perhaps indicating dust-free environments (Bouwens et al. 2010a), and their stellar masses have been inferred from measurements in the rest-frame optical (González et al. 2010; Labbé et al. 2010a, 2010b). These observations inform theoretical models of galaxy formation and attempt to probe the amount of radiation available to affect the ionization state of the intergalactic medium (IGM) but are often limited by survey sensitivity. The traditional interpretation of such data relies on an assumed ratio of UV luminosity to star formation rate (SFR) that requires burst ages longer than the exponential burst timescale and timescales longer than $1 \mathrm{Gyr}$ (Madau et al. 1998). This assumption cannot be satisfied at redshifts $z>6$, where the age of the universe is shorter than $1 \mathrm{Gyr}$.

Many theoretical studies based on numerical and semianalytic techniques, have shown that the luminosity function (LF) of high-redshift LBGs can be explained by the hierarchical formation of dark matter halos whose associated baryonic gas forms stars in merger-generated bursts (e.g., Baugh et al. 2005; Finlator et al. 2011; Lacey et al. 2011; Salvaterra et al. 2010). Analytic work has tried to fit simpler models to the observed LF to probe the duty cycle and mass-to-light ratio of observed galaxies (e.g., Stark et al. 2007; Trenti et al. 2010) assigning a single galaxy luminosity for each halo mass, while others have considered that the mass of a host halo may merely define the probability distribution from which a galaxy's luminosity is drawn (e.g., Cooray \& Milosavljević 2005a, 2005b; Cooray \& Ouchi 2006; Vale \& Ostriker 2004, 2006, 2008). Associating the mass of underlying halos with observed luminosity is crucial for describing the clustering properties and bias of high-redshift galaxies as well as the contribution of cosmic variance to fluctuations in the measured abundance from field to field (e.g., Trenti \& Stiavelli 2008; Muñoz \& Loeb 2008a; Overzier et al. 2009; Muñoz et al. 2010; Robertson 2010a, $2010 b)$. The relationship may also provide insights into how much ionizing radiation is provided by galaxies too faint to be detected with current instruments.

The amount of currently undetected UV radiation at high redshifts is unknown. While the Early Release Science observations with WFC3/IR can probe down to 27.5 AB mag (e.g., Bouwens et al. 2010c), it is almost certain that many fainter galaxies remain to be observed by the James Webb Space Telescope (JWST; e.g., Barkana \& Loeb 2000b; Wyithe \& Loeb 2006; Salvaterra \& Ferrara 2006). Galaxies should exist in halos down to a mass below which the assembly or retainment of gas is suppressed. While these dwarf galaxies near the suppression limit may be faint, their abundance may make them, in aggregate, large contributors to the total UV background. The exact suppression mass of galaxy formation is due to an unknown combination of the heating of the IGM during reionization and thermal and mechanical feedback by internal mechanisms such as supernovae (Wyithe \& Loeb 2006).

We propose that the suppression of galaxy formation below a certain halo mass threshold may already be evident from the currently observed LFs at $z \gtrsim 6$. Because bright galaxies are formed primarily through mergers among fainter ones and since the contribution to the population of bright galaxies from lower mass halos shining at the bright end of their luminosity distributions need not be negligible, we expect the decrease in the faint end of the LF due to the suppression of galaxy formation to be gradual and extend to larger luminosities than previously anticipated. We couple hierarchical merger trees to a simple model of star formation to calculate the luminosity distribution function (LDF) for galaxies as a function of their host halo mass and the resulting LF. The suppression mass of galaxy formation applied to the variety of merger histories provides us with a physically motivated explanation for the fraction of halos that are forming stars at the time of observation (i.e., the duty cycle) as a function of mass. We calculate the amount of unobserved UV radiation at $z=6,7$, and 8 and test the applicability of the Madau et al. (1998) relationship between UV luminosity and 
SFR. We note that Raičević et al. (2011) recently considered the effect of photoionization on the LF using the GALFORM model of galaxy formation (Cole et al. 2000; Baugh et al. 2005), but our models produce very different results at the faintest luminosities. This is likely because photoionization in the GALFORM model only affects gas cooling while allowing already cold gas to form stars even in the smallest halos.

In Section 2, we describe the merger trees, star formation model, and suppression prescriptions used to populate the LDF for each halo mass. We describe the resulting shape and mass dependence of the LDF in Section 3 and discuss the physical origin of the luminous duty cycle of halos in Section 4. In Section 5, we use analytic fits to our numerical calculations of the LDF and duty cycle to probe the mean of the LDFs, the resulting star formation efficiency, and the mass at which star formation is suppressed by matching the observed LFs at $z \gtrsim 6$. In Section 6, we compare the SFR to the prediction from the UV luminosity. We then discuss the implications for the ionization state of the IGM of the suppressed star formation in low-mass halos and the abundance of faint galaxies yet to be observed in Section 7. Finally, we summarize our main conclusions in Section 8 .

\section{THE MODEL}

Our calculation of the luminosity of high-redshift galaxies has two main components: a merger-tree builder and a star formation model. Throughout, we assume a flat, $\Lambda$ CDM cosmology with cosmological parameters taken from the WMAP-5 data release (Komatsu et al. 2009; Dunkley et al. 2009).

\subsection{Merger Histories}

We generate merger trees based on the extended Press-Schechter procedure outlined in Somerville \& Kolatt (1999). The method selects, for each descendent, a series of progenitors from the mass-weighted conditional mass function truncated at an upper mass limit for each subsequently selected progenitor such that the total mass in progenitors does not exceed the descendent mass. Once the difference in mass between the descendent and the growing list of progenitors falls below $M_{\text {res }}$, the resolution limit of the algorithm, the remaining mass is assigned as diffuse accretion. If a descendent has two or more progenitors, we determine the merger ratio by considering the two largest progenitors. If the mass ratio between the two largest progenitors is smaller than $1: 3$, we denote the interaction to be a major merger; all other configurations are minor mergers. We have tested our procedure using a threshold ratio of 1:7 and found no noticeable difference in our results. The algorithm is then iterated on each progenitor until the tree is ended when the masses of all progenitors have fallen below $M_{\text {res }}$.

\subsection{Starbursts}

Each halo in the merger tree is assumed initially to contain an amount of baryonic gas equal to a fixed fraction $\left(\Omega_{b} / \Omega_{m}\right)$ of its halo mass. This gas is gradually converted into stars through bursts of star formation. There is a great body of evidence that the starbursts that illuminate LBGs are generated in mergers rather than in a quiescent mode (e.g., Baugh et al. 2005; Lacey et al. 2011). Therefore, we ignore all quiescent star formation and generate starbursts exclusively during major mergers.

After a minor merger, all starbursts taking place in associated branches of the tree are allowed to continue simultaneously with their own reservoirs of gas. If not fully coalesced at the time of observation, these simultaneous bursts may appear as multiple cores in the galaxy morphology or simply be beyond our current ability to resolve (Oesch et al. 2010). However, in a major merger, all of these bursts are shut off and a new one is begun. The gas remaining from all progenitors is assumed to be instantaneously funneled to the center where it forms a new disk. Following Mo et al. (1998) and Barkana \& Loeb (2000a), we assume the disk to have an exponential shape such that the surface density falls off as $\Sigma=\Sigma_{0} e^{-r / R_{\mathrm{d}}}$. At high redshift, when the energy density of the universe is dominated by the contribution from matter, the corresponding exponential size scale of the disk is given by

$$
\begin{aligned}
R_{\mathrm{d}}= & \frac{1}{\sqrt{2}}\left(\frac{j_{\mathrm{d}}}{m_{\mathrm{d}}}\right) \lambda r_{\mathrm{vir}} \approx 0.1 h^{-1} \mathrm{kpc}\left(\frac{\lambda}{0.05}\right)\left(\frac{j_{\mathrm{d}}}{m_{\mathrm{d}}}\right) \\
& \times\left(\frac{v_{\mathrm{c}}}{30 \mathrm{~km} \mathrm{~s}^{-1}}\right)\left[\frac{H(z=7)}{H_{0}}\right]^{-1},
\end{aligned}
$$

where we take the specific angular momentum of the disk to be equal to that of the halo (i.e., $j_{\mathrm{d}} / m_{\mathrm{d}}=1$ ), $r_{\mathrm{vir}}$ is the halo virial radius, $v_{\mathrm{c}}$ is the circular velocity of the halo, $H$ is the Hubble parameter, and $\lambda$ is the spin parameter which we draw randomly for each disk from a log-normal distribution centered at $\bar{\lambda}=0.05$ with a standard deviation $\sigma_{\lambda}=0.5$ in log-space. The central surface density is $\Sigma_{0}=M_{\text {gas }} / 2 \pi R_{\mathrm{d}}^{2}$.

Following Kennicutt (1998), the surface star formation rate density is given by $\Sigma_{\mathrm{SFR}}=\epsilon \Sigma_{\text {gas }} / t_{\text {dyn }}$, where $\epsilon$ is the fraction of gas converted to stars in a dynamical time $t_{\text {dyn }}$. We verified that the disks under consideration are unstable to fragmentation (i.e., have a Toomre $Q$-parameter smaller than unity). Using surface densities averaged over the exponential scale radius of the disk and $t_{\mathrm{dyn}}=2 \pi R_{\mathrm{d}} / v_{\mathrm{c}}, \epsilon$ is found empirically to be $\sim 20 \%$. However, this relation also holds in azimuthallyaveraged rings at radius $r$ with $t_{\mathrm{dyn}}=2 \pi r / v_{\mathrm{c}}$. Since we are interested in the total SFR-produced entire disk from gas added at any radius, we integrate through the disk considering separately the contributions inside and outside the exponential scale radius:

$$
\begin{aligned}
\dot{M}_{\star} & =\dot{M}_{\star}\left(<R_{\mathrm{d}}\right)+2 \pi \int_{R_{\mathrm{d}}}^{\infty} r \Sigma_{\mathrm{SFR}}(r) d r \\
& =\epsilon \Sigma_{0} v_{\mathrm{c}}\left[\left(\int_{R_{\mathrm{d}}}^{\infty} \frac{r e^{-r / R_{\mathrm{d}}}}{R_{\mathrm{d}}} d r\right)+\left(\int_{R_{\mathrm{d}}}^{\infty} e^{-r / R_{\mathrm{d}}} d r\right)\right] \\
& =\epsilon \Sigma_{0} v_{\mathrm{c}} R_{\mathrm{d}}\left[\left(e^{-1}\right)+\left(1-2 e^{-1}\right)\right] .
\end{aligned}
$$

Substituting for $R_{\mathrm{d}}$ and $\Sigma_{0}$, we find

$$
\begin{aligned}
\dot{M}_{\star} \approx & 0.66 \mathrm{M}_{\odot} \mathrm{yr}^{-1}\left(\frac{M_{\mathrm{gas}}}{10^{8} h^{-1} M_{\odot}}\right)\left(\frac{\epsilon}{0.2}\right) \\
& \times\left(\frac{\lambda}{0.05}\right)^{-1}\left[\frac{H}{H(z=7)}\right] .
\end{aligned}
$$

Since $\dot{M}_{\star}=-\dot{M}_{\text {gas }}$ in a single burst between major mergers, Equation (3) represents a differential equation in $M_{\star}$ (or $M_{\mathrm{gas}}$ ) whose solution is an exponential with a timescale given by

$$
\tau \approx 0.27 t_{\mathrm{age}}(z)\left(\frac{\epsilon}{0.2}\right)^{-1}\left(\frac{\lambda}{0.05}\right)
$$


where

$$
t_{\text {age }}(z) \approx 0.52 h^{-1} \mathrm{Gyr}\left(\frac{1+z}{8}\right)^{-3 / 2}
$$

is the age of the universe at redshift $z$.

In each time step, we use results from a simple stellar population generated by Starburst99 (Leitherer et al. 1999) to calculate the contribution of each newly added group of stars to the final luminosity at $1500 \AA$. We assume a Salpeter initial mass function (IMF) with a slope of 2.35 between 1 and $100 M_{\odot}$ and a metallicity $4 \%$ of solar.

Finally, we include the effect of a suppression in galaxy formation below halos of a given mass. Some combination of processes, such as supernovae feedback or photoionization, may push or heat the gas so that it escapes the gravitational potential well of the halo. Thus, no starbursts will be generated in halos smaller than $M_{\text {supp }}$, and neither can a halo smaller than $M_{\text {supp }}$ be a constituent in a starburst-generating major merger even if the resulting halo is larger than $M_{\text {supp }}$. These two conditions (but especially the latter) combine to make inactive even some halos larger than $M_{\text {supp }}$ (see Section 4). The simplest way to incorporate these effects into our model is simply to set $M_{\text {res }}=M_{\text {supp }}$ in generating the merger trees. This prescription is not quite realistic, since the feedback processes that suppress star formation are undoubtedly time dependent, especially during reionization. However, for simplicity, we assume that the contributions to the LF from minihalos smaller than $M_{\text {supp }}$ before reionization are minimal by the redshifts considered here. For convenience, we define

$$
\begin{array}{r}
m_{\mathrm{h}} \equiv \log _{10}\left(M_{\mathrm{h}} / M_{\odot}\right), \\
m_{\text {supp }} \equiv \log _{10}\left(M_{\text {supp }} / M_{\odot}\right) .
\end{array}
$$

\section{THE LUMINOSITY DISTRIBUTION FUNCTION}

Our model results in an approximately log-normal distribution for the UV galaxy luminosities (1500 ̊) produced by halos of a given mass:

$$
\frac{d P}{d \log _{10} L}=\frac{1}{\sqrt{2 \pi \sigma_{\mathrm{L}}^{2}}} \exp \left(-\frac{\left(\log _{10}\left(L / L_{\mathrm{c}}\right)\right)^{2}}{2 \sigma_{\mathrm{L}}^{2}}\right)
$$

in agreement with previous assumptions (e.g., Cooray \& Milosavljević 2005a, 2005b; Cooray \& Ouchi 2006; Vale \& Ostriker 2004, 2006, 2008). As anticipated by the self-similarity of halo mergers (Fakhouri et al. 2010), we find a roughly linear relationship between the characteristic luminosity $L_{\mathrm{c}}$ and halo mass (up to $10^{12} M_{\odot}$ ) independent of redshift. Bouwens et al. (2008) previously estimated a power-law slope of 1.24 based on Bouwens et al. (2007) data at $z \sim 4$. We reiterate that many of the assumptions that went into our model, including the lack of a quiescent component to star formation and dust extinction, are only valid at redshifts beyond 6 . We do not find a change in the proportionality of luminosity to halo mass at high masses as considered by Bouwens et al. (2008). Since the timescale for the coalescence of subhalos after the merger is related to the ratio of their masses (Wetzel et al. 2009; Wetzel \& White 2010) and we have selected major merges based on a fixed mass ratio, we do not expect a falloff in the rate of major mergers in more massive halos. Finally, we also find $\sigma_{\mathrm{L}}$ between 0.2 and 0.3 for all masses and redshifts considered. Consequently, the galactic luminosity produced by halos of the same mass can easily vary by $\sim 1.5$ mag or more. For convenience, we will assume an analytic expression for the LDF as a function of halo mass given by a log-normal distribution with $L_{\mathrm{c}}$ proportional to $M_{\mathrm{h}}$ and a fixed value of $\sigma_{\mathrm{L}}=0.25$ for all calculations of the LF throughout the rest of this paper.

\section{THE LUMINOUS DUTY CYCLE OF HALOS}

We first clarify a slight ambiguity of definition in the literature. The duty cycle, $\epsilon_{\mathrm{DC}}$, is defined as the fraction of a halo's lifetime over which it is luminous. If halos fluctuate stochastically on and off, then $\epsilon_{\mathrm{DC}}$ also represents the probability that a halo will be on at the moment of observation and the fraction of all halos at that time that are on. Considering halos of a given mass for which a single luminosity has been assumed, this concept of the duty cycle lowers the abundance of halos observed from that predicted in the halo mass function, i.e.,

$$
\frac{d n_{\mathrm{obs}}(L)}{d L}=\epsilon_{\mathrm{DC}} \frac{d n}{d M_{\mathrm{h}}} \frac{d L\left(M_{\mathrm{h}}\right)}{d M_{\mathrm{h}}} .
$$

In various models, $\epsilon_{\mathrm{DC}}$ may be a function of variables such as mass or redshift or left as a constant parameter.

However, if the luminosity of a halo or the frequency of its being in a luminous state is not constant in time, the fraction of observable halos for a given halo mass need not be equal to $\epsilon_{\mathrm{DC}}$. In our model, there are two reasons why a halo may not be observable. The first is that, given the continuous distribution in luminosity for a given halo mass, some are not bright enough to be detectable at their distance. However, these halos will simply appear in another luminosity bin, and we will proceed by first calculating the full LF and subsequently applying an observable threshold for a given survey. The second reason why a halo may not be observable is because, in the limited history of the universe at the moment of observation, its merger tree does not contain a single major merger whose constituents were more massive than $M_{\text {supp }}$. Thus, according to our model, it will not have had even one starburst, and will remain completely dark. Halos much more massive than $M_{\text {supp }}$ have had at least one starburst-generating merger, while those closer in mass to $M_{\text {supp }}$ may not have since many of its recent progenitors are below $M_{\text {supp }}$. We define the probability that a halo of a given mass has had at least one starburst-generating merger as the "active fraction," $\epsilon_{\mathrm{AF}}$.

Using our merger-tree code, we find a relation for the active fraction of halos as a function of mass that is nearly independent of redshift. Since $\epsilon_{\mathrm{AF}}$ depends only on the distribution of merger histories, it is also independent of $\epsilon$ and the other details of our star formation model in Section 2.2. We show, in Figure 1, $\epsilon_{\mathrm{AF}}$ as a function of mass calculated from our code for several values of $m_{\text {supp }}$. Each point was generated using enough random merger histories to produce at least 100 active galaxies. A good fitting formula for $\epsilon_{\mathrm{AF}}$ is given by

$$
\log _{10} \epsilon_{\mathrm{AF}}\left(m_{\mathrm{h}}, m_{\text {supp }}\right)=-\frac{1}{8\left(m_{\mathrm{h}}-m_{\text {supp }}\right)^{2.6}},
$$

where $m_{\mathrm{h}}$ and $m_{\text {supp }}$ are defined by Equation (6). Throughout the rest of this paper, we rely on Equation (9) to compute $\epsilon_{\mathrm{AF}}$ for continuous ranges of $m_{\mathrm{h}}$ and $m_{\text {supp }}$. Our results show that the abundance of halos hosting galaxies is suppressed even for halo masses up to an order of magnitude larger than the suppression threshold. As we will see in Section 5, the large range of suppression masses combines with the distribution of 


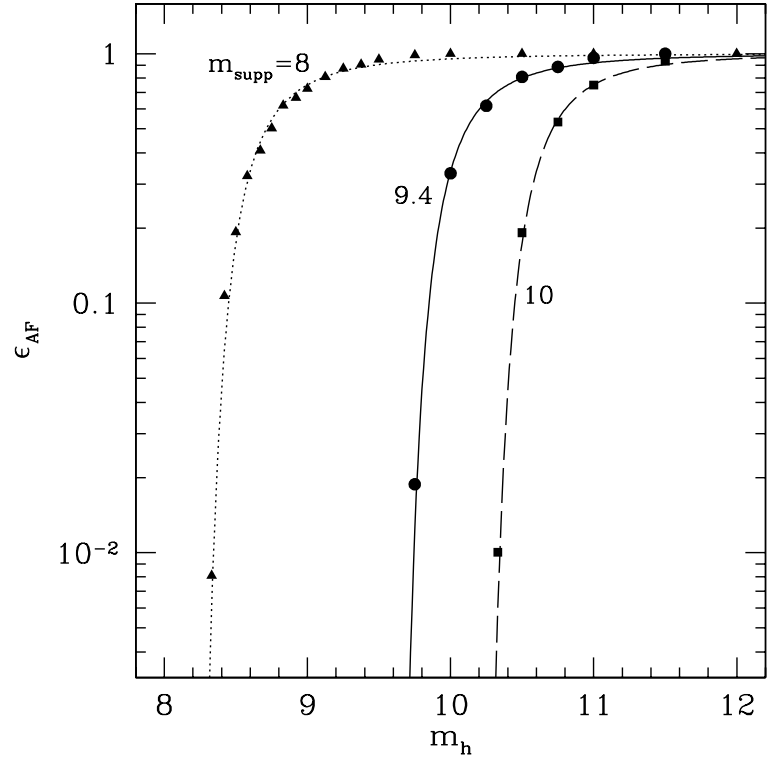

Figure 1. Active fraction, $\epsilon_{\mathrm{AF}}$, or the fraction of halos that have had at least one starburst-generating merger in their lifetimes as a function of halo mass, $m_{\mathrm{h}}$, for three different values of the galaxy formation suppression threshold mass $M_{\text {supp. }}$ Squares, circles, and triangles show merger-tree simulation results for $m_{\text {supp }}=8,9.4$, and 10, respectively, while dotted, solid, and long-dashed lines show the results from Equation (9) for the same suppression mass values. Enough merger histories we generated so that each point represents at least 100 active galaxies.

possible luminosities for each halo to result in a gentle cutoff at the faint end of the LF rather than a sudden drop at a critical luminosity. However, our model naturally reproduces a high value of $\epsilon_{\mathrm{AF}}$ for the largest halo masses, consistent with the rapid evolution of the halo mass function at $z \gtrsim 6$ (Trenti et al. 2010).

\section{FITTING THE LUMINOSITY FUNCTION}

We model the LF of high-redshift galaxies using the halo mass function of Sheth \& Tormen (2002) and the analytic prescriptions for the LDF and $\epsilon_{\mathrm{AF}}$ derived in Sections 3 and 4. We fit our model LFs to the data available at $z=6$ from Bouwens et al. (2007) and recent samples of more than 60 $z \sim 7$ and nearly $50 z \sim 8$ LBGs from Bouwens et al. (2010c) adopting the same magnitude conventions and ignoring, for simplicity, any bright-end upper limits. All magnitudes we reference in this paper are rest-frame UV absolute magnitudes in the AB system. We have calculated LFs at single redshifts for comparison with observations, ignoring for the time being the mass-dependent distribution of galaxies over the photometric redshift range of high-redshift surveys and its effects on the LF (Muñoz \& Loeb 2008b). At each redshift, we allow two fit parameters: $L_{10}=L_{\mathrm{c}}\left(M_{\mathrm{h}}=10^{10} M_{\odot}\right)$ and $M_{\text {supp }} L_{10}$ is directly related to the star formation efficiency $\epsilon$ in our model for fixed choices of metallicity and IMF. In Figure 2, we plot the minimum reduced chi-squared, $\chi_{\text {red }}^{2}$ (i.e., chi-squared per degree of freedom), values matching the observed LF at $z=6,7$, and 8 as a function of $M_{\text {supp }}$. Values of $L_{10}$ have been calculated to minimize $\chi_{\text {red }}^{2}$ for each value of $M_{\text {supp }}$.

A minimum in $\chi_{\text {red }}^{2}$ appears at $m_{\text {supp }} \approx 9.5$ at $z=6, \approx 9.4$ at $z=7$, and $\approx 9.42$ at $z=8$. At $z=6$, all combinations of $L_{10}$ and $M_{\text {supp }}$ are ruled out at the $70 \%$ level. However, values of $m_{\text {supp }}<8.55$ and $>9.7$ are ruled out at the $95 \%$ level, while $m_{\text {supp }}>9.8$ is ruled out with $99 \%$ confidence. At $z=7$ and

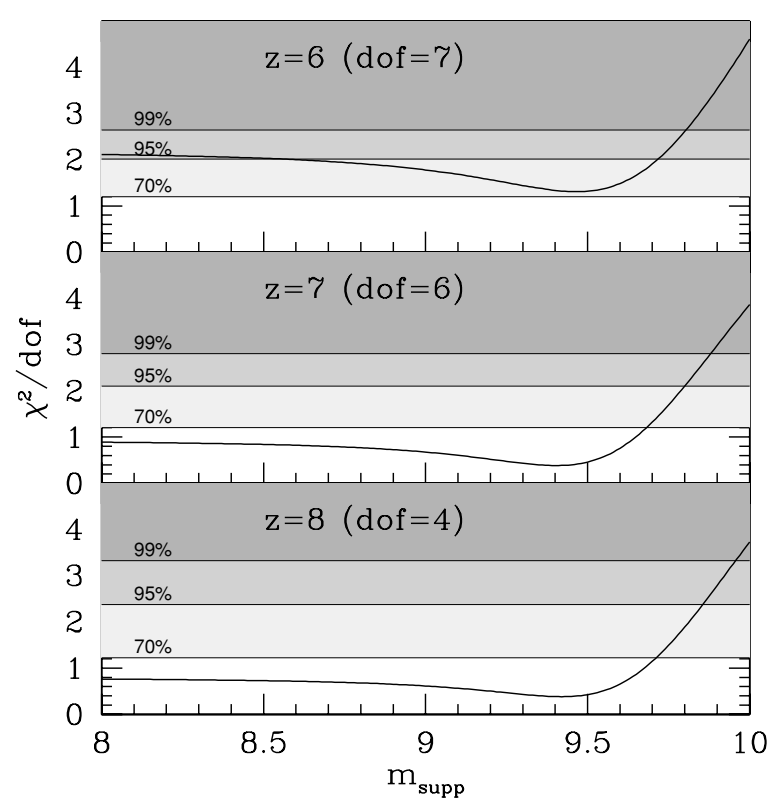

Figure 2. Minimum reduced chi-squared (i.e., chi-squared per degree of freedom) marginalized over $L_{10}$ as a function of $m_{\text {supp }}$. The top, middle, and bottom panels show fits to the data at $z=6,7$, and 8 , respectively, while the shaded regions in each panel denote parameter space rejected with $70 \%, 95 \%$, and $99 \%$ confidence.

$z=8$, no constraints are placed on the minimum value of $m_{\text {supp }}$ at the $70 \%$ level or stronger. However, $m_{\text {supp }}>9.7(9.7),>9.8$ (9.85), and >9.9 (9.95) are ruled out with 70\%, 95\%, and $99 \%$ confidence, respectively, at $z=7$ (8).

These results clearly indicate that, while the masses of halos hosting observed LBGs are typically thought to be $>10^{10} M_{\odot}$, lower luminosity galaxies must exist in halos smaller than $10^{10} M_{\odot}$, corresponding to a virial temperature of about $2 \times 10^{5} \mathrm{~K}$, and very likely in ones at least as small as $5 \times 10^{9} M_{\odot}$ $\left(10^{5} \mathrm{~K}\right)$. They also tentatively suggest that the minimum mass halo capable of hosting galaxies may be around $2.5 \times 10^{9} M_{\odot}$ $\left(7 \times 10^{4} \mathrm{~K}\right)$ with halos less massive than about $3.5 \times 10^{8} \mathrm{M}_{\odot}$ $\left(1.7 \times 10^{4} \mathrm{~K}\right)$ unable to host galaxies with some confidence given the data at $z=6$.

Chi-squared is minimized when $L_{10} \approx 27.2,27.4$, and 27.7 at $z=6,7$, and 8 , respectively. For our choices of metallicity and IMF, these values imply that galaxy formation is relatively inefficient, with very small fractions of galactic gas $(0.2 \%$, $0.4 \%$, and $0.5 \%$ for each redshift) being converted into stars per dynamical time.

Our best-fit LFs, along with ones assuming $m_{\text {supp }}=8,9$, and 10, are shown for each redshift in Figure 3. The data from Bouwens et al. $(2007,2010 \mathrm{c})$ are plotted for comparison. The best-fit model deviates qualitatively from a Schechter function fit outside the observed magnitude range. At the bright end, for magnitudes $<-21$, our predicted LF remains much flatter than a Schechter fit, which is already beginning to drop exponentially. The shallower slope is due to two effects: first, the exponential tail of the halo mass function falls off more slowly with increasing mass than a Schechter function with luminosity proportional to mass, and second, the large spread in the luminosity permitted for each halo mass allows abundant, smaller halos emitting at higher than average luminosity to bolster the population of bright galaxies.

On the other hand, the suppression of star formation drastically reduces the abundance of galaxies at magnitudes fainter than currently observable compared with expectations from a 


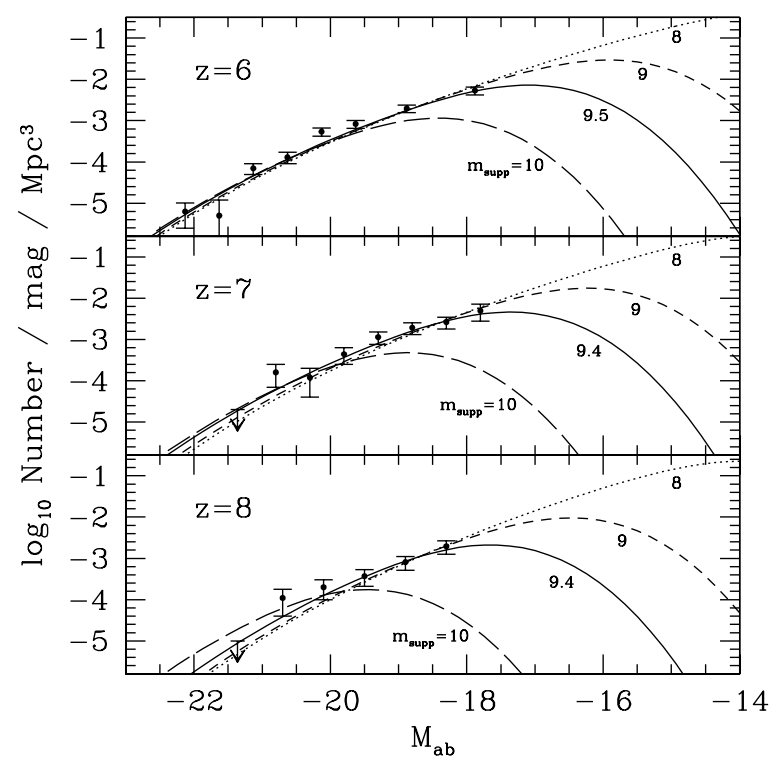

Figure 3. Comparison of our best-fit LFs to the data. The top, middle, and bottom panels display results for $z=6,7$, and 8 , respectively. The points and error bars mark observations from Bouwens et al. (2007, 2010c). Dotted, short-dashed, and long-dashed curves are LFs assuming $m_{\text {supp }}=8$, 9, and 10, respectively, with the best-fit value of $L_{10}$ for each value of $m_{\text {supp }}$. Finally, solid lines show results with the absolute minimum value of chi-square at each redshift. The best-fit values of $m_{\text {supp }}$ are $9.47,9.4$, and 9.42, for $z=6,7$, and 8 , respectively.

simple extrapolation of the Schechter function. The result is a flatter LF slope in the observed region for increasing $m_{\text {supp }}$. Figure 3 clearly illustrates the disparate predictions for the abundance of faint galaxies between different fiducial values of $M_{\text {supp }}$. Additional data at only about a magnitude fainter than the current observational threshold will greatly improve our ability to constrain the minimum halo mass capable of forming galaxies. For reference, observations down to a magnitude of -16.8 at $z=7$ will require a sensitivity of about $1.5 \mathrm{nJy}$, close to what is expected with JWST. However, while the $1 \sigma$ errors in the current data were calculated based on the shot noise and cosmic variance from an amalgam of observations from several different fields, we conservatively estimate the $1 \sigma$ error on the abundance at this magnitude in a single $2^{\prime} \times 2^{\prime}$ pointing of NIRCam on JWST to be about $50 \%$ (Muñoz et al. 2010).

We have explicitly ignored the influence of quiescent star formation in our model. Such a mechanism in small halos may reduce the effects that we describe of a galaxy suppression mass on the faint end of the LF making it more difficult to probe such physics with future surveys. However, work by Lacey et al. (2011) has shown that merger-driven starbursts do dominate the UV LF down to at least magnitude -17 at $z=6$ and to -15 by $z=10$, albeit with a very different IMF. Thus, while more complicated models may be required for highprecision measurements of $M_{\text {supp }}$ even with a complete LF, we are confident that deeper surveys in the not-too-distant future will help illuminate some of the physics of galaxy suppression.

\section{STAR FORMATION RATE}

The SFR of high-redshift galaxies is important for understanding the star formation history of the universe (Madau et al. 1998) and the ionization state of the IGM (Madau et al. 1999). Its estimation relies on a proportionality between UV luminosity and SFR based on two assumptions: an exponential burst of star formation has a timescale, $\tau$, that is longer than $1 \mathrm{Gyr}$, and the
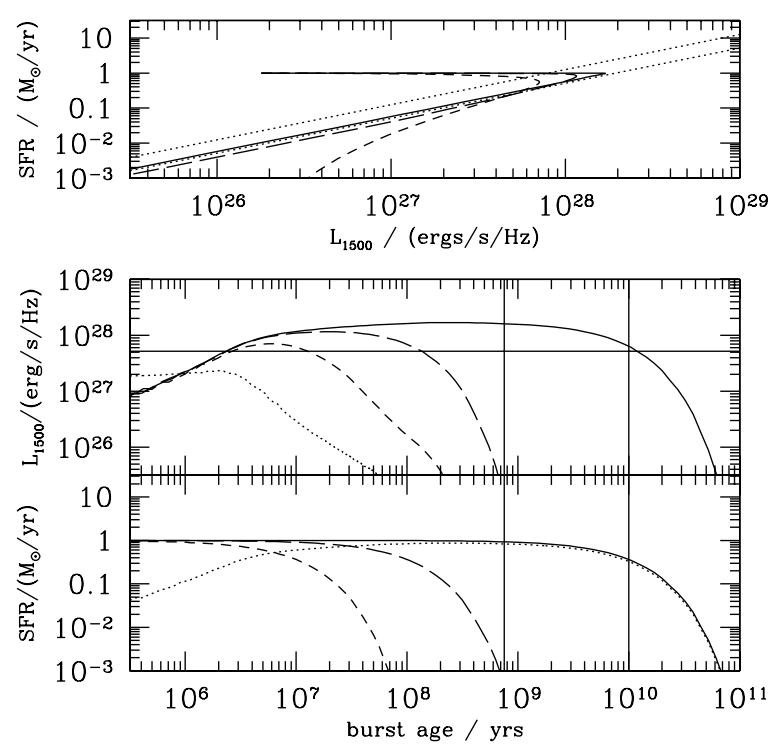

Figure 4. UV luminosity and SFR evolution of exponential bursts with $\left(\tau / \mathrm{Gyr}, \dot{M}_{\star}(t=0) \mathrm{M}_{\odot}{ }^{-1} \mathrm{yr}\right)=(10,1),(0.1,3)$, and $(0.01,10)$ denoted by solid, long-dashed, and short-dashed curves, respectively. The top panel tracks the bursts in SFR- $L_{1500}$ space. Here, the upper and lower dotted lines show a proportional relationship between SFR and $L_{1500}$ with constants of $8 \times 10^{27} \mathrm{erg} \mathrm{s}^{-1} \mathrm{~Hz}^{-1}\left(\mathrm{M}_{\odot} \mathrm{yr}^{-1}\right)^{-1}$ and $2 \times 10^{28} \mathrm{erg} \mathrm{s}^{-1} \mathrm{~Hz}^{-1}\left(\mathrm{M}_{\odot} \mathrm{yr}^{-1}\right)^{-1}$, respectively. The middle panel shows the burst light curves with right and left vertical lines denoting the $\tau=10 \mathrm{Gyr}$ and the age of the universe at $z=7$, respectively. The light curve for an instantaneous burst producing $10^{6} M_{\odot}$ worth of stars is given by the dotted curve. The horizontal line marks the observable threshold at a magnitude of -18 . The bottom panel plots the evolution of the SFR with time, while the dotted curve here shows the SFR expected from the relationship between SFR and $L_{1500}$ given the burst luminosity as a function of time.

stellar population is observed after one exponential timescale has past (Madau et al. 1998). However, if the age of the universe is shorter than $1 \mathrm{Gyr}$, at least one of these assumptions must be violated.

For the best-fit star formation efficiencies we found from the data at $z=6,7$, and 8 that the typical exponential starburst timescale given by Equation (5) is of order $\tau \sim 10 \mathrm{Gyr}$, an order of magnitude or more longer than the age of the universe. Equation (3) gives the typical SFR to be of order $1 M_{\odot} \mathrm{yr}^{-1}$ in a burst with $10^{10} M_{\odot}$ worth of gas remaining; if the amount of initial gas in a halo as a fraction of the total halo mass is $\Omega_{\mathrm{b}} / \Omega_{\mathrm{m}} \approx 0.16$, this corresponds to the initial SFR in a halo of about $6.25 \times 10^{10} \mathrm{M}_{\odot}$.

Figure 4 shows the evolution of the luminosity at $1500 \AA$, $L_{1500}$, and SFR, $\dot{M}_{\star}$, with time and their relationship calculated for exponential bursts using a simple stellar population from Starburst99 (Leitherer et al. 1999). Solid lines represent typical bursts in high-redshift galaxies with the initial SFR set at $1 M_{\odot} \mathrm{yr}^{-1}$ and the exponential timescale $\tau=10 \mathrm{Gyr}$. For $t>\tau$, both $\dot{M}_{\star}$ and $L_{1500}$ decrease exponentially over time with timescale $\tau$ so that $L_{1500}$ is proportional to $\dot{M}_{\star}$. This is because the exponential timescale is much longer than the lifetime of the stars that dominate the UV luminosity. The amplitude of the relation is set by the IMF and metallicity of the stellar population; for the choices described in Section 2.2, we find approximately $L_{1500}=2 \times 10^{28}\left(\dot{M}_{\star} / \mathrm{M}_{\odot} \mathrm{yr}\right) \mathrm{erg} \mathrm{s}^{-1} \mathrm{~Hz}^{-1}$ with a proportionality constant a factor of 2.5 different from the $8 \times 10^{27} \mathrm{erg} \mathrm{s}^{-1} \mathrm{~Hz}^{-1}\left(\mathrm{M}_{\odot} \mathrm{yr}^{-1}\right)^{-1}$. However, before $t=\tau$, the luminosity is still rising with increasing time, while the SFR remains essentially unchanged. Since the age of the universe is 
much less than $\tau$, all bursts are observed in this phase before the $L_{1500}-\dot{M}_{\star}$ proportionality has stabilized.

Thus, the SFR will typically be somewhat higher than that inferred from the $L_{1500}-\dot{M}_{\star}$ proportionality. The ratio between the true and expected SFRs will depend on how close the burst is to its maximum luminosity, the point where the expected SFR is approximately equal to its initial value. The burst light curve is relatively flat near its maximum value over the time approximately $10^{7}-10^{9} \mathrm{yr}$ after it begins. If the burst is more than $10^{7} \mathrm{yr}$ old at the time of observation, the difference between the true and expected SFRs will not be very significant. A burst observed at $z=7$ is $10^{7} \mathrm{yr}$ old if it started at $z \approx 7.07$.

For completion, we also show in Figure 4 the evolution of $L_{1500}$ and $M_{\star}$ for bursts with $\tau=0.1$ and $0.01 \mathrm{Gyr}$, less than the 1 Gyr minimum considered by Madau et al. (1998). These timescales are achieved at $z=7$ if for combinations of the star formation efficiency and the disk spin parameter such that $\epsilon^{-1} \lambda=0.17$ and 0.017 , respectively. The luminosity in each case begins to decline before reaching the maximum it would have achieved had $\tau$ been longer. The falloff in luminosity is only slightly slower than exponential for $\tau=0.1 \mathrm{Gyr}$ so that the SFR and UV luminosity reach a nearly proportional relationship after $t=\tau$, albeit with a coefficient slightly higher than that seen for higher $\tau$. However, the decline in luminosity is more power law than exponential for $\tau=0.01 \mathrm{Gyr}$ leading to a very nonlinear relationship between SFR and luminosity after $t=\tau$. In both cases, the SFR is much less than expected for a given luminosity. This is because the timescale $\tau$ is not so much longer than the lifetimes of the stars that dominate the UV luminosity. Luminosity from stars produced at $t=\tau$, for example, contribute significantly to the luminosity at $0.1 \tau$, whereas the contribution would be completely negligible for much larger $\tau$. Consequently, the luminosity for a given instantaneous SFR can be much higher than expected.

Using our merger tree and star formation code, we calculate the instantaneous $\dot{M}_{\star}$ at the time of observation for each of our modeled galaxies and test the accuracy of the Madau et al. (1998) proportionality at $z=7$ over a wide range of halo masses and for a full distribution of spin parameters and merger histories. Figure 5 shows the relationship between instantaneous SFR, $\dot{M}_{\star}$, and UV luminosity, $L_{1500}$, for galaxies in halos at $z=7$ with masses of $10^{10}, 10^{10.5}, 10^{11}$, and $10^{11.5} M_{\odot}$. We have set $m_{\text {supp }}=9.4$. Each point represents a single halo, and where more than one ongoing starburst is present, we have simply added the contributing SFRs. The discrete "lines" of points above the main body for the smaller halo masses is a resolution effect of our code; while their exact positions should not be taken as precise, such points do represent a population of halos lying above the standard $L_{1500}-\dot{M}_{\star}$ relation.

The majority of points in Figure 5 do show a rough proportionality between $L_{1500}$ and $\dot{M}_{\star}$. However, the proportionality constant is slightly higher than the $2 \times$ $10^{28} \mathrm{erg} \mathrm{s}^{-1} \mathrm{~Hz}^{-1}\left(\mathrm{M}_{\odot} \mathrm{yr}^{-1}\right)^{-1}$ value for bursts with ages longer than their exponential timescale with the difference depending on halo mass. Lower mass halos tend to be populated by younger bursts that are further from reaching their maximum luminosity than higher mass halos. If we constrain $L_{1500} \propto \dot{M}_{\star}$, we find a proportionality constant of $1.7 \times 10^{28} \mathrm{erg} \mathrm{s}^{-1} \mathrm{~Hz}^{-1}\left(\mathrm{M}_{\odot} \mathrm{yr}^{-1}\right)^{-1}$ for $10^{10} M_{\odot}$ halos which estimates SFRs to be about $15 \%$ higher than the constant for older bursts. Given the typical uncertainties in measuring the total SFR at high redshift-sample completeness, cosmic variance, uncertain IMF and metallicity, etc.-an

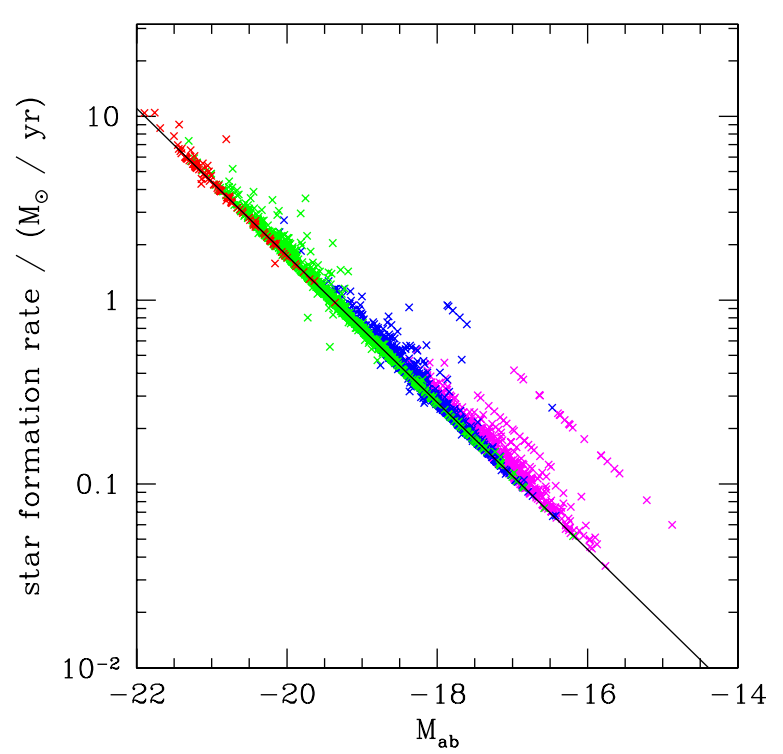

Figure 5. SFR vs. rest-frame UV magnitude of simulated halos. Magenta, blue, green, and red points denote halos of mass $10^{10}, 10^{10.5}, 10^{11}$, and $10^{11.5} M_{\odot}$, respectively. The solid, black line marks $L_{1500}=2 \times 10^{28}$ $\left(\dot{M}_{\star} / \mathrm{M}_{\odot} \mathrm{yr}\right) \mathrm{erg} \mathrm{s}^{-1} \mathrm{~Hz}^{-1}$.

additional $\sim 20 \%$ error will not significantly affect current estimates.

\section{IONIZATION STATE OF THE IGM}

After cosmic reionization, the ionization state of the IGM depends on the balance between the recombination rate and the production rate of ionizing photons. On its own, the formation of stars in galaxies can maintain the ionization of the IGM through the production of ionizing photons if the star formation rate density (SFRD) is higher than a critical value given by Madau et al. (1999):

$$
\dot{\rho}_{\star} \approx 2 \times 10^{-3} f_{\mathrm{esc}}^{-1} C\left(\frac{1+z}{10}\right)^{3} \mathrm{M}_{\odot} \mathrm{yr}^{-1} \mathrm{Mpc}^{-3},
$$

where $f_{\text {esc }}$ is the fraction of ionizing photons produced in galaxies that escape into the IGM and $C$ is the IGM clumping factor.

Using the standard $L_{1500}$ to $\dot{M}_{\star}$ conversion, recent observational studies have estimated the currently observable SFRD to be just enough to keep the universe ionized if $f_{\mathrm{esc}}^{-1} C=1$. However, much of the star formation below the observable threshold is not included. In Figure 6, we compare our calculations for the total SFRD at $z=6,7$, and 8 assuming the best-fit values of $L_{10}$ and $M_{\text {supp }}$ at each redshift to the observed estimates and to Equation (10). We show results for the $L_{1500}$ to $\dot{M}_{\star}$ ratios of both $8 \times 10^{27} \mathrm{erg} \mathrm{s}^{-1} \mathrm{~Hz}^{-1}\left(\mathrm{M}_{\odot} \mathrm{yr}^{-1}\right)^{-1}$ (the typically used value) and $2 \times 10^{28} \mathrm{erg} \mathrm{s}^{-1} \mathrm{~Hz}^{-1}\left(\mathrm{M}_{\odot} \mathrm{yr}^{-1}\right)^{-1}$ (consistent with our choices of IMF and metallicity). We also show the factor by which the total values of the SFRD and UV luminosity exceed those observed by Bouwens et al. (2007) and Bouwens et al. (2010c). Factors less than unity indicate that the observed points are higher than average in the universe at that redshift due to Poisson fluctuations or cosmic variance so that the observed SFRD is higher than the average over the whole population.

Our results show that the ability of the total galaxy population to account for the UV background required to keep the IGM ionized depends critically on the value of $m_{\text {supp }}$. For $m_{\text {supp }}=8$, the total SFRD or UV luminosity is about 3-9 times the observed 

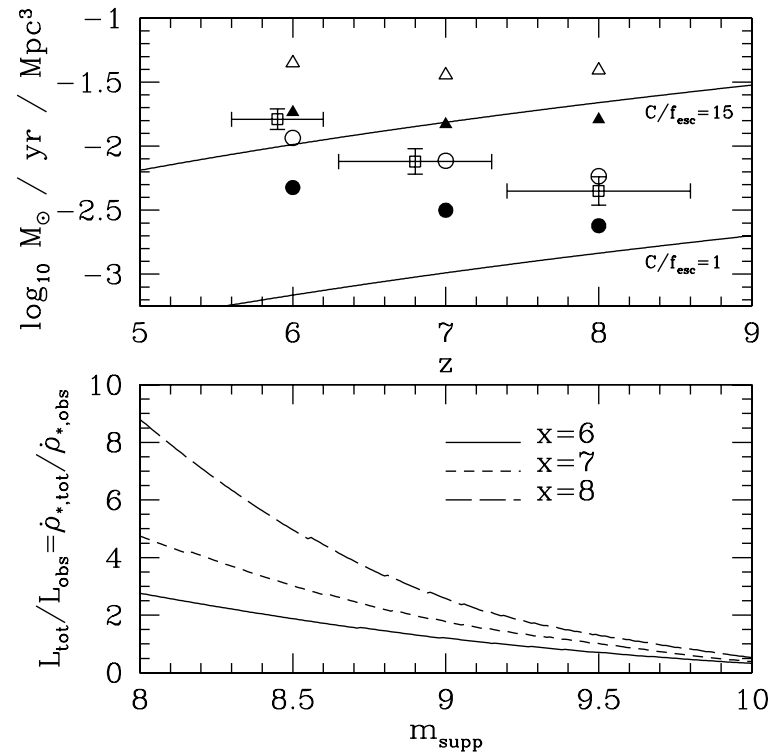

Figure 6. Top panel shows the SFRD produced by the total galaxy population at $z=6,7$, and 8 . Circles denote results using best-fit values of $M_{\text {supp }}$ and $L_{10}$ at each redshift, while triangles assume $M_{\text {supp }}=10^{8} M_{\odot}$ and the corresponding best-fit values of $L_{10}$. Filled (empty) points use $L_{1500} / \dot{M}_{\star}=$ $2 \times 10^{28}\left(8 \times 10^{27}\right) \mathrm{erg} \mathrm{s}^{-1} \mathrm{~Hz}^{-1}\left(\mathrm{M}_{\odot} \mathrm{yr}^{-1}\right)^{-1}$. Square points with error bars denote observed values from Bouwens et al. (2007) and Bouwens et al. (2010c). The minimum SFRD required to keep the IGM ionized as given by Equation (10) for $f_{\mathrm{esc}}^{-1} C=15$ and 1 are shown by the upper and lower solid lines, respectively. The bottom panel shows the ratio of the total UV luminosity or SFRD to the Bouwens et al. (2007) and Bouwens et al. (2010c) observations as a function of $M_{\text {supp}}$. The solid, short-dashed, and long-dashed lines denote $z=6,7$, and 8, respectively.

values with more star formation and luminosity missing at higher redshift. However, at the best-fit values of $m_{\text {supp }}=9.5$ at $z=6$ and 9.4 at $z=7$ and 8 , the galaxy population produces no more SFRD than observed (and somewhat less for $z=6$ ). Assuming $f_{\mathrm{esc}}^{-1} C=1$, the total SFRD for all parameters and redshifts considered meet the requirement for maintaining the ionization of the IGM. However, if $f_{\text {esc }}^{-1} C=15$ (e.g., $f_{\text {esc }}=0.2$ and $C=3$ ), the galaxy population can keep the IGM at $z=8$ ionized only for a choice of IMF and metallicity that gives the Madau et al. (1998) ratio of $L_{1500}$ to $\dot{M}_{\star}$ ratio of $3 \times 10^{27} \mathrm{erg} \mathrm{s}^{-1} \mathrm{~Hz}^{-1}\left(\mathrm{M}_{\odot} \mathrm{yr}^{-1}\right)^{-1}$ and if $m_{\text {supp }} \sim 8$, lower than our best-fit value. Finally, since the amount of star formation below the observable limit increases with redshift, we find that the evolution of the total SFRD with redshift is much flatter than that observed.

\section{CONCLUSIONS}

In this paper, we combine a standard merger-tree algorithm with a simple star formation prescription designed to encapsulate the main physical processes relevant at $z \gtrsim 6$. Our model both accounts for a range of possible galaxy luminosities for each halo mass and includes a sharp galaxy formation cutoff in halo mass below $M_{\text {supp }}$.

1. We confirm that the luminosity distribution function for halos of a given mass is a roughly log-normal distribution with a variance of $\sim 1.5 \mathrm{mag}$ and a proportional relationship between the mean luminosity and halo mass (see Section 3 ).

For metallicity $4 \%$ of solar and a Salpeter IMF, we find the normalizations for the mean log-luminosities at a fixed halo mass of $10^{10} M_{\odot}$ to be $\log _{10}\left(L_{10} \mathrm{erg}^{-1} \mathrm{~s} \mathrm{~Hz}\right)=27.2$,
27.4 , and 27.7 at $z=6,7$, and 8, respectively, suggesting that for a fixed halo mass, galaxies are brighter on average at higher redshift, consistent with results from the Schechter fits. However, while the exponential tail of the high-redshift halo mass function is less sharp than that of a Schechter function, the range of possible luminosities for a fixed halo mass further slows the falloff of the predicted galaxy LF at the bright end. While still being consistent with the data, our shallower LF anticipates the discovery of a larger population of very bright galaxies at $z=7$ and 8 as survey fields increase in size with abundances consistent with recent studies by Ouchi et al. (2009) and Castellano et al. (2010).

2. We also show that an active fraction of halos that approaches unity with increasing halo mass can be naturally explained by a suppression halo mass for galaxy formation combined with the variety of possible merger histories (see Section 4).

This active fraction is well approximated by the formula given in Equation (9). One can easily use this expression, along with our log-normal distributions of UV luminosity for each halo mass to calculate the galaxy LF from the halo mass function. The resulting LF does not have a sharp cutoff at the faint end but rather turns over gently. Thus, we predict that, as long as future observations show an LF that increases with ever decreasing luminosity, the surveyed region will never be volume-complete.

3. The current data suggest that the minimum mass halo capable of hosting galaxies may be around $2.5 \times 10^{9} \mathrm{M}_{\odot}$, corresponding to a virial temperature of $7 \times 10^{4} \mathrm{~K}$ (see Section 5).

We find a strong upper limit of $M_{\text {supp }}<6 \times 10^{9} M_{\odot}$ $\left(10^{5} \mathrm{~K}\right)$ with at least $95 \%$ confidence. However, lower limits from the current data are quite weak with halos less massive than about $3.5 \times 10^{8} M_{\odot}\left(1.7 \times 10^{4} \mathrm{~K}\right)$ unable to host galaxies with some confidence given the data at $z=6$.

4. We find a best-fit star formation efficiency at high redshift of approximately $0.2 \%-0.5 \%$ per dynamical time, implying a starburst exponential timescale much longer than the age of the universe.

A more top-heavy IMF would have required even less efficient star formation, corresponding to even longer burst timescales, to produce the same observed luminosities. However, the long burst timescale does not create lightbulblike galaxies that, once switched on, are always emitting with the same luminosity. Instead continued merger activity disrupts old bursts and replaces them with new ones based on the particular history of the host halo.

5. We show that the proportionality of $L_{1500}$ to $\dot{M}_{\star}$ is usually an adequate approximation (see Section 6).

While the Madau et al. (1998) proportionality relies on long-lived bursts in the tail of their exponentially decreasing rate of star formation, most bursts at $z=7$ are emitting near their maximum luminosity where the track of SFR versus UV luminosity begins to join the proportional relationship. Despite their young ages compared to their exponential timescale, this is because the bursts are typically older than $10^{7} \mathrm{yr}$ at the time of observation, old enough that the massive stars providing the bulk of the UV luminosity are beginning to die out as fast as new ones are added. Although the lowest mass halos may host very young bursts that have somewhat higher SFRs than expected for their luminosities, using a standard proportionality of $L_{1500}$ to $\dot{M}_{\star}$ adds additional errors of only tens of 
percent. However, some care must be taken in selecting a constant of proportionality consistent with specific choices of metallicity and IMF rather than using the Madau et al. (1998) value indiscriminately. Additionally, since bursts are likely to remain close to their maximum SFRs and luminosities for most of their lifetimes, ongoing accretion between major mergers is less likely to be important.

6. When extrapolated down to faint luminosities below the current observable threshold, the total SFRD of the galaxy population will be only at most 3-9 times higher than what has already been observed even if the minimum halo mass forming galaxies is as low as $10^{8} M_{\odot}$ (see Section 7).

The gentle turnover at the faint end of the LF, even given a sharp cutoff in the halo mass capable of producing galaxies, results in less star formation below the observable limit than if the LF dropped sharply at the mean luminosity corresponding to the same halo mass. While the total galaxy population with $m_{\text {supp }}=8$ may be able to keep the IGM ionized given $f_{\text {esc }}^{-1} C \sim 15$, for our best-fit value of $m_{\text {supp }} \approx 9.4$, no significant star formation lies below a rest-frame UV magnitude of -18 . In such a case, galaxies may only be responsible for maintaining the ionization state of the IGM if $f_{\mathrm{esc}}^{-1} C \sim 1$. Interestingly, we also find that, since the amount of missing star formation increases with redshift, the redshift evolution of the total star formation history of the universe is flatter than observed.

Although the current data from LBG dropouts do not place a strong lower limit on the minimum halo mass required to host galaxies at redshifts $\gtrsim 6$, we have shown that JWST and other future deep surveys will provide much tighter constraints. These results will not only shed light on the contribution of galaxies to the UV background that keeps the IGM ionized but also hint at the feedback physics that limits galaxy formation.

We thank Steve Furlanetto and Michele Trenti for useful comments and discussions. This work was supported in part by NSF grant AST-0907890 and NASA grants NNX08AL43G and NNA09DB30A (for A.L.).

\section{REFERENCES}

Barkana, R., \& Loeb, A. 2000a, ApJ, 531, 613

Barkana, R., \& Loeb, A. 2000b, ApJ, 539, 20

Baugh, C. M., Lacey, C. G., Frenk, C. S., Granato, G. L., Silva, L., Bressan, A., Benson, A. J., \& Cole, S. 2005, MNRAS, 356, 1191

Bouwens, R. J., Illingworth, G. D., Franx, M., \& Ford, H. 2007, ApJ, 670, 928
Bouwens, R. J., et al. 2008, ApJ, 686, 230

Bouwens, R. J., et al. 2009, arXiv:0912.4263

Bouwens, R. J., et al. 2010a, ApJ, 708, L69

Bouwens, R. J., et al. 2010b, ApJ, 709, L133

Bouwens, R. J., et al. 2010c, arXiv: 1006.4360

Bunker, A. J., et al. 2010, MNRAS, 409, 855

Castellano, M., et al. 2010, A\&A, 524, A28

Cole, S., Lacey, C. G., Baugh, C. M., \& Frenk, C. S. 2000, MNRAS, 319, 168

Cooray, A., \& Milosavljević, M. 2005a, ApJ, 627, L85

Cooray, A., \& Milosavljević, M. 2005b, ApJ, 627, L89

Cooray, A., \& Ouchi, M. 2006, MNRAS, 369, 1869

Dunkley, J., et al. 2009, ApJS, 180, 306

Fakhouri, O., Ma, C., \& Boylan-Kolchin, M. 2010, MNRAS, 406, 2267

Finkelstein, S. L., Papovich, C., Giavalisco, M., Reddy, N. A., Ferguson, H. C., Koekemoer, A. M., \& Dickinson, M. 2010, ApJ, 719, 1250

Finlator, K., Oppenheimer, B. D., \& Davé, R. 2011, MNRAS, 410, 1703

González, V., Labbé, I., Bouwens, R. J., Illingworth, G., Franx, M., Kriek, M., \& Brammer, G. B. 2010, ApJ, 713, 115

Kennicutt, R. C., Jr. 1998, ApJ, 498, 541

Komatsu, E., et al. 2009, ApJS, 180, 330

Labbé, I., et al. 2010a, ApJ, 708, L26

Labbé, I., et al. 2010b, ApJ, 716, L103

Lacey, C. G., Baugh, C. M., Frenk, C. S., \& Benson, A. J. 2011, arXiv:1004.3545

Leitherer, C., et al. 1999, ApJS, 123, 3

Madau, P., Haardt, F., \& Rees, M. J. 1999, ApJ, 514, 648

Madau, P., Pozzetti, L., \& Dickinson, M. 1998, ApJ, 498, 106

McLure, R. J., Dunlop, J. S., Cirasuolo, M., Koekemoer, A. M., Sabbi, E., Stark, D. P., Targett, T. A., \& Ellis, R. S. 2010, MNRAS, 403, 960

Mo, H. J., Mao, S., \& White, S. D. M. 1998, MNRAS, 295, 319

Muñoz, J. A., \& Loeb, A. 2008a, MNRAS, 385, 2175

Muñoz, J. A., \& Loeb, A. 2008b, MNRAS, 386, 2323

Muñoz, J. A., Trac, H., \& Loeb, A. 2010, MNRAS, 405, 2001

Oesch, P. A., et al. 2010, ApJ, 709, L21

Ouchi, M., et al. 2009, ApJ, 706, 1136

Overzier, R. A., Guo, Q., Kauffmann, G., De Lucia, G., Bouwens, R., \& Lemson, G. 2009, MNRAS, 394, 577

Raičević, M., Theuns, T., \& Lacey, C. 2011, MNRAS, 410, 775

Robertson, B. E. 2010a, ApJ, 713, 1266

Robertson, B. E. 2010b, ApJ, 716, L229

Salvaterra, R., \& Ferrara, A. 2006, MNRAS, 367, L11

Salvaterra, R., Ferrara, A., \& Dayal, P. 2010, arXiv:1003.3873

Sheth, R. K., \& Tormen, G. 2002, MNRAS, 329, 61

Somerville, R. S., \& Kolatt, T. S. 1999, MNRAS, 305, 1

Stark, D. P., Loeb, A., \& Ellis, R. S. 2007, ApJ, 668, 627

Trenti, M., \& Stiavelli, M. 2008, ApJ, 676, 767

Trenti, M., Stiavelli, M., Bouwens, R. J., Oesch, P., Shull, J. M., Illingworth, G. D., Bradley, L. D., \& Carollo, C. M. 2010, ApJ, 714, L202

Vale, A., \& Ostriker, J. P. 2004, MNRAS, 353, 189

Vale, A., \& Ostriker, J. P. 2006, MNRAS, 371, 1173

Vale, A., \& Ostriker, J. P. 2008, MNRAS, 383, 355

Wetzel, A. R., Cohn, J. D., \& White, M. 2009, MNRAS, 395, 1376

Wetzel, A. R., \& White, M. 2010, MNRAS, 403, 1072

Wyithe, J. S. B., \& Loeb, A. 2006, Nature, 441, 322

Yan, H., Windhorst, R. A., Hathi, N. P., Cohen, S. H., Ryan, R. E., O’Connell, R. W., \& McCarthy, P. J. 2010, Res. Astron. Astrophys., 10, 867 